\title{
Nociceptive Memory in the Brain: Cortical Mechanisms of Chronic Pain
}

\author{
Ming Yi and Haolin Zhang \\ Neuroscience Research Institute, Peking University, Beijing 100191, China \\ Review of Eto et al.
}

In neurobiology, learning refers to changed responses to the same stimulus, whereas the retention of these changes is memory. These terms encompass a variety of neuronal and behavioral processes, including chronic pain. Chronic pain persists after an injury has healed and results from significant functional and structural changes in the nervous system similar to memory processes. As a result, chronic pain has been proposed to be "a persistence of the memory of pain and/or the inability to extinguish the memory of pain evoked by an initial inciting injury" (Apkarian et al., 2009).

Where and how is this nociceptive memory stored? This is a complicated question covering multiple levels of the pain pathway, from peripheral nociceptors to the cerebral cortex. It is generally agreed that the cerebral cortex plays a significant role in pain perception and modulation. Researchers have identified the "pain matrix," a group of brain regions consistently activated by acute pain. The primary (S1) and secondary (S2) somatosensory cortices belong to the lateral pain pathway, which is involved in the sensory

Received June 28, 2011; revised July 29, 2011; accepted July 30, 2011.

M.Y. was funded by the China Postdoctoral Science Foundation (20090460180). We thank You Wan for helpful comments on this manuscript and the entire Wan laboratory for insightful discussions.

The authors declare no competing financial interests.

Correspondence should be addressed to either Ming Yi or Haolin Zhang, Neuroscience Research Institute, Peking University, 38 Xueyuan Road, Beijing 100191, China. E-mail: mingyi@bjmu.edu.cn or zhanghaolin@ bjmu.edu.cn.

DOI:10.1523/JNEUROSCI.3279-11.2011

Copyright $(\underset{2}{ } 2011$ the authors $\quad 0270-6474 / 11 / 3113343-03 \$ 15.00 / 0$ dimension of pain, whereas the anterior cingulate cortex (ACC) is part of the medial pathway, which is important for pain affection (Brooks and Tracey, 2005). ACC responds to persistent nociceptive stimulation with significant plasticity, which contributes to the maintenance of chronic pain (Li et al., 2010). Whether S1 undergoes similar changes and acts as a site of chronic pain storage is unclear.

A recent study addressed this issue with imaging, electrophysiological, and behavioral experiments (Eto et al., 2011). In this study, the authors first examined whether chronic pain changed neuronal activities in S1. In a mouse model of chronic inflammatory pain induced by intraplantar injections of Complete Freund's adjuvant (CFA), two-photon $\mathrm{Ca}^{2+}$ imaging and patch-clamp recordings in S1 layer $2 / 3$ (L2/3) showed that both spontaneous activity and activity induced by sensory stimulation were significantly increased relative to control mice.

The authors next examined potential multiregional interactions in chronic pain by injecting CNQX, an AMPA receptor antagonist, into the S1 of CFA-treated mice. This treatment significantly decreased the slope of field EPSPs in the ACC resulting from electrical stimulation of the hindpaw. Such changes were not observed in control mice, suggesting that these interregional changes were specific to chronic pain. Disrupting these changes by microinjecting CNQX into either $\mathrm{S} 1$ or ACC of CFA-treated mice significantly attenuated mechanical allodynia.
Overall, the study by Eto et al. suggests that S1 neurons, like those in the ACC, show significant plasticity associated with the development of chronic pain, and these changes contribute to pain maintenance. These findings, together with data from other studies, support the idea that chronic pain can be understood as a nociceptive memory, and they suggest several directions of future research.

The finding that S1 neurons increase their activity under chronic pain conditions is consistent with neuroimaging studies in humans (Brooks and Tracey, 2005). Thus, S1 neurons have apparently "memorized" the chronic pain. As mentioned earlier, S1 is consistently activated in acute pain and encodes the somatosensory dimension of pain. Does the hyperactivity of S1 neurons under chronic pain states represent a simple continuum of acute pain, or is it distinct? Unfortunately, Eto et al. examined only one time point ( 7 $\mathrm{d}$ after CFA injection) and did not examine S1 activity in the acute phase of the model (e.g., within $24 \mathrm{~h}$ of pain initiation). Nonetheless, their pharmacological and electrophysiological experiments indirectly answered this question. Intra-S1 injection of CNQX significantly increased the paw-withdrawal threshold of CFAinjected mice but not control mice, suggesting that AMPA receptors in the $\mathrm{S} 1$ selectively participate in processing peripheral stimuli under chronic pain states but not under acute, physiological pain. In addition, the increased amplitudes of EPSCs from L2/3 pyramidal neurons 
evoked by electrical stimulation of the L4 region in CFA-treated but not control mice indicated intra-S1 remodeling in chronic pain. These conclusions are supported by a study showing differential roles of $\mathrm{S} 1$ in acute and chronic pain (Wang et al., 2009).

How does the activity of S1 neurons change during the transition from acute to chronic pain? We have little evidence to address this question at present, but one might imagine that it is a gradual procedure associated with various neuroplastic changes, analogous to the transition from short-term to long-term memory. Indeed, nearly all changes identified in other forms of memory have been reported to be associated with the development of chronic pain, from functional potentiation to structural rearrangement (Apkarian et al., 2009; Metz et al., 2009). A tool that might clarify how the transition happens is multiunit recording in awake animals, which has been widely used in studies of hippocampal learning, but rarely in chronic pain studies. This method not only enables long-term monitoring of neuronal activity in the same subject, but it also allows more natural experimental conditions without anesthesia-induced effects on cortical activity (Ramani and Wardhan, 2008).

The most important finding in Eto and colleagues' study is the interregional interactions in chronic pain: hyperactivity of S1 the L2/3 neurons appears to exacerbate pain behavior by facilitating ACC activity. What connects S1 and ACC in chronic pain? One obvious pathway is projections from S1 to S2 and insular cortex, which in turn project to the ACC. As discussed earlier, chronic pain induction results in synaptic plasticity in S1 and ACC, which might alter dynamics of the network. Alternatively, synchronized brain oscillations, whose role in cognition has been intensively studied, may participate in chronic pain as well. During spatial memory tasks, coherent theta oscillations have been reported between the hippocampus and several brain regions, including the entorhinal cortex, the prefrontal cortex, and the striatum (for review, see Düzel et al., 2010). It is suggested that labile information initially encoded in the hippocampus is transmitted to the neocortex for long-term storage by coherent spiking of large populations of neurons during these oscillations in subsequent immobility and slow-wave sleep (Buzsáki, 1996). This hypothesis has gained much experimental support in recent years: selective disruption of sharp wave/ripple complexes in the hippocampus-entorhinal cortex during the consolidation period causes performance impairment in rats trained on a spatial memory task (Girardeau et al., 2009). In other words, coordinated oscillations are not merely manifestations of dynamic multiregional interactions, but may have significant roles in information encoding and storage. In pain, peripheral nociceptive stimulation increased phase locking between theta and gamma band responses (Babiloni et al., 2002; Wang et al., 2011), whereas patients with chronic neurogenic pain exhibit excess EEG oscillation in the $4-9 \mathrm{~Hz}$ theta frequency band (Stern et al., 2006) and high thalamocortical theta coherence (Sarnthein and Jeanmonod, 2008). However, the exact role of these oscillations in the maintenance of chronic pain is unclear. Simultaneous neuronal recording in several regions combined with pharmacological interventions will be of great value in answering this question.

A significant feature of learning and memory is the correlated structural, functional, and behavioral changes (Cacucci et al., 2008). Persistent pain results in significant structural and functional changes in the nervous system (Apkarian et al., 2009). Can these changes return to normal when the source of pain is eliminated? An early study reported that S1 reorganization associated with phantom limb pain could be reversed shortly after the elimination of pain by regional blockade (Birbaumer et al., 1997). More recently, Seminowicz et al. (2011) showed that patients with chronic lower back pain (CLBP) had decreased cortical thickness in several regions, including the left dorsolateral prefrontal cortex (DLPFC), left S1, and right ACC. Successful treatment of these patients was associated with increased cortical thickness in the left DLPFC but not other regions, suggesting that structural changes in chronic pain are at least partially reversible. However, it was unclear why other atrophic areas, including S1 and ACC, failed to recover. One possibility is that atrophy in these regions is irreversible, whereas an alternative explanation is that the follow-up time is too short (6 months after successful treatment vs 5 years' CLBP). Understanding these questions will benefit the prediction of therapeutic outcomes.

Finally, a firm conclusion in the neurobiology of learning and memory is that different types of memory have distinct mechanisms (e.g., declarative memory vs procedural memory). A similar distinction can be made in pain: various chronic pain states have distinct central mechanisms (Apkarian et al., 2009). Eto et al. performed their experiments in a chronic inflammatory pain model. But whether the same conclusions can be drawn in models of chronic neuropathic or cancer pain is unknown. A very recent study has reported structural and functional plasticity in S1 associated with neuropathic pain (Kim and Nabekura, 2011), suggesting at least partial similarities between different chronic pain conditions. Further investigation is required to clarify the identical and distinct mechanisms under various pain states.

In summary, accumulating evidence suggests that chronic pain is a type of nociceptive memory. Evidence from Eto et al.'s study further supports the idea that pain is a network phenomenon mediated not by single proteins or brain regions, but by multiple pathways at cortical, subcortical, spinal, and peripheral levels. Nonetheless, more research is necessary to understand how this nociceptive memory is encoded and stored, as well as to find appropriate ways to eradicate it without affecting normal physiological functions.

\section{References}

Apkarian AV, Baliki MN, Geha PY (2009) Towards a theory of chronic pain. Prog Neurobiol 87:81-97.

Babiloni C, Babiloni F, Carducci F, Cincotti F, Rosciarelli F, Arendt-Nielsen L, Chen AC, Rossini PM (2002) Human brain oscillatory activity phase-locked to painful electrical stimulations: a multi-channel EEG study. Hum Brain Mapp 15:112-123.

Birbaumer N, Lutzenberger W, Montoya P, Larbig W, Unertl K, Töpfner S, Grodd W, Taub E, Flor H (1997) Effects of regional anesthesia on phantom limb pain are mirrored in changes in cortical reorganization. J Neurosci 17:5503-5508.

Brooks J, Tracey I (2005) From nociception to pain perception: imaging the spinal and supraspinal pathways. J Anat 207:19-33.

Buzsáki G (1996) The hippocampo-neocortical dialogue. Cereb Cortex 6:81-92.

Cacucci F, Yi M, Wills TJ, Chapman P, O’Keefe J (2008) Place cell firing correlates with memory deficits and amyloid plaque burden in Tg2576 Alzheimer mouse model. Proc Natl Acad Sci U S A 105:7863-7868.

Düzel E, Penny WD, Burgess N (2010) Brain oscillations and memory. Curr Opin Neurobiol 20:143-149.

Eto K, Wake H, Watanabe M, Ishibashi H, Noda M, Yanagawa Y, Nabekura J (2011) Interregional contribution of enhanced activity of the primary somatosensory cortex to the anterior cingulate cortex accelerates chronic pain behavior. J Neurosci 31:7631-7636.

Girardeau G, Benchenane K, Wiener SI, Buzsáki G, Zugaro MB (2009) Selective suppression of hippocampal ripples impairs spatial memory. Nat Neurosci 12:1222-1223.

Kim SK, Nabekura J (2011) Rapid synaptic remodeling in the adult somatosensory cortex 
following peripheral nerve injury and its association with neuropathic pain. J Neurosci 31:5477-5482.

Li XY, Ko HG, Chen T, Descalzi G, Koga K, Wang H, Kim SS, Shang Y, Kwak C, Park SW, Shim J, Lee K, Collingridge GL, Kaang BK, Zhuo M (2010) Alleviating neuropathic pain hypersensitivity by inhibiting PKMzeta in the anterior cingulate cortex. Science 330:1400-1404.

Metz AE, Yau HJ, Centeno MV, Apkarian AV, Martina M (2009) Morphological and functional reorganization of rat medial prefrontal cortex in neuropathic pain. Proc Natl Acad Sci U S A 106:2423-2428.

Ramani R, Wardhan R (2008) Understanding anesthesia through functional imaging. Curr Opin Anaesthesiol 21:530-536.

Sarnthein J, Jeanmonod D (2008) High thalamocortical theta coherence in patients with neurogenic pain. Neuroimage 39: 1910-1917.

Seminowicz DA, Wideman TH, Naso L, Hatami-Khoroushahi Z, Fallatah S, Ware MA, Jarzem P, Bushnell MC, Shir Y, Ouellet JA, Stone LS (2011) Effective treatment of chronic low back pain in humans reverses abnormal brain anatomy and function. J Neurosci 31:7540-7550.

Stern J, Jeanmonod D, Sarnthein J (2006) Persistent EEG overactivation in the cortical pain matrix of neurogenic pain patients. Neuroimage 31:721-731

Wang J, Li D, Li X, Liu FY, Xing GG, Cai J, Wan Y (2011) Phase-amplitude coupling between theta and gamma oscillations during nociception in rat electroencephalography. Neurosci Lett 499:84-87.

Wang N, Wang JY, Luo F (2009) Corticofugal outputs facilitate acute, but inhibit chronic pain in rats. Pain 142:108-115. 